Fundamentally, however, I came away from Toxic Exposures confused as to its intended audience. The liberal use of didactic terms and constructs that presume fluency in the language and literature of medical sociology is likely to make the text too dense for most lay readers; there are also occasional passages that are overly doctrinaire and likely to put off even an otherwise receptive health scientist.
At one point in the text, Brown supports a seven-point manifesto of 'critical epidemiology', with the fifth point being: "Include in research reporting the explicit discussion of assumptions, values, and the social construction of scientific knowledge." This is certain to bewilder epidemiologists, most of whom see themselves as engaged scientists already bothered by a governmental policy-making apparatus that is overly politicized in the opposite direction. Fifty years on, it seems as though the two cultures are farther apart than ever. Paul D. Blanc is professor of occupational and environmental medicine at the University of California, San Francisco, California 94143-0924, USA and is the author of How Everyday Products Make People Sick: Toxins at Home and in the Workplace.

\title{
Celebrating four centuries of astronomy
}

\author{
The Telescope: Its History, Technology \\ and Future \\ by Geoff Andersen \\ Princeton University Press: 2007. 256 pp. \\ $\$ 29.95$
}

\section{René Racine}

The International Year of Astronomy in 2009 will mark 400 years of astronomical discovery, pioneered by Galileo Galilei in Padua, Italy. The anniversary will stimulate interest in the tools that made these discoveries possible, so a flush of new books on telescopes is likely to appear and sell briskly. Geoff Andersen's timely work also arrives at a point when astronomers and engineers are starting to construct a new generation of revolutionary instruments for ground-based and spacebased astronomy.

Andersen's book covers the historical and technological evolution of the telescope. It is written for a broad readership and "avoids, wherever possible, the gruesome details which keep so many of us employed". It will be appreciated by laypersons and experts for its clarity and, in places, the insight it provides into the optics, engineering and, indeed, scientific purposes and limitations of telescopes.

The book is enriched by the perspective it offers on remote sensing and surveillance applications, a field that the author has contributed to as a research physicist at the US Air Force Academy. This enables him to develop some interesting speculation on the capabilities of extant 'spy telescopes'.

Historical notes are exact, lively and generally up-to-date. Witness the recent discovery of the full name of Laurent Cassegrain - seventeenth-century inventor of the Cassegrain telescope - uncovered by André Baranne and Françoise Launay in the Journal of Optics in 1997, and not generally known even to experts. References to current projects, such as the forthcoming Hubble Space Telescope service mission, the Thirty Meter Telescope partnership and the 100-metre-aperture OWL (for 'overwhelmingly large') telescope, are inevitably dated, given the rapid evolution of the field.

Professional astronomers and experienced amateur telescope-makers might be tempted to skip the introductory chapters on optics,

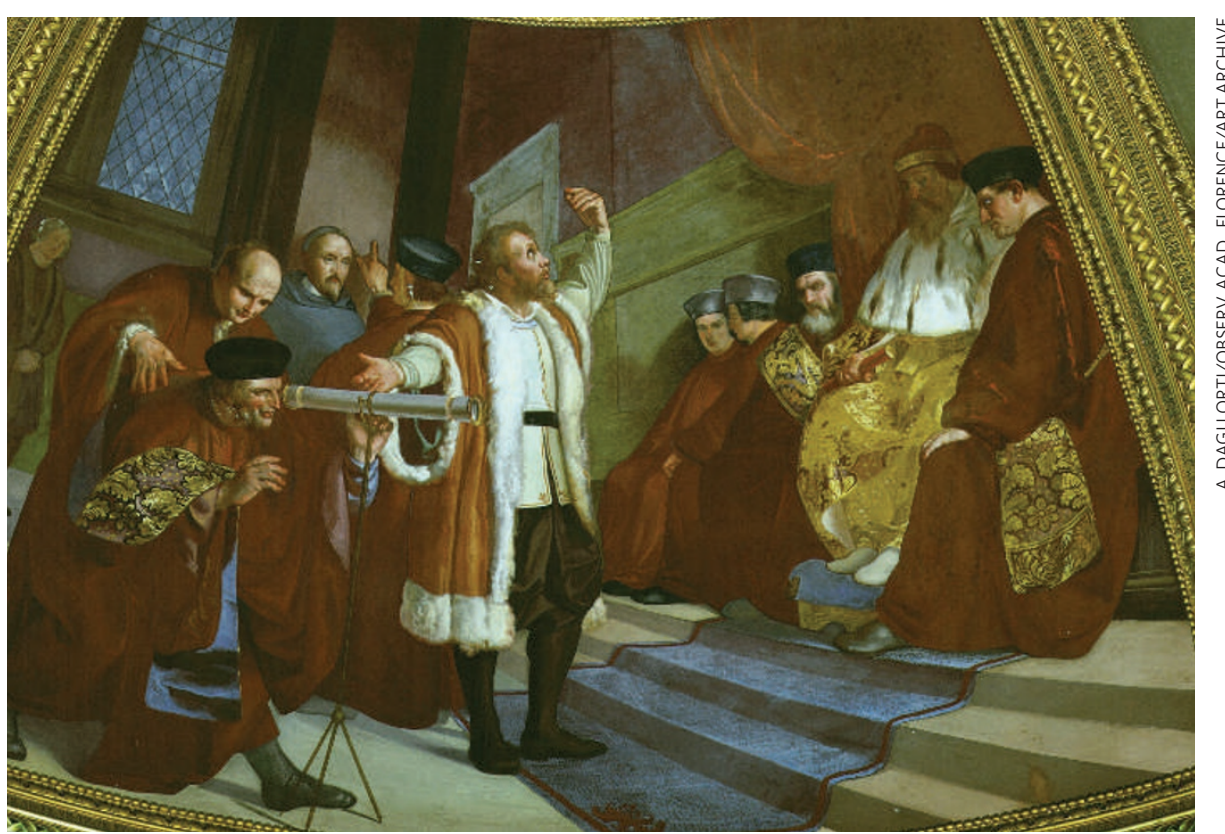

Galileo presents the most powerful telescope of its time to the Doge and Senate of Venice in 1609.

the basics of telescope design, light-analysis instruments and observing-site properties, but it would be at the cost of missing some pedagogical gems. These include the quasiintuitive explanation of Poisson's 'bright spot', which vindicated the theory of diffraction and the wave nature of light.

Informative chapters on interferometry and advanced telescope techniques - active and adaptive optics, laser guide stars, laser communications, and so on - are seldom found in such detail in similar works, adding to the value of the book. The analogies and differences in telescopes operating in various domains, from $\gamma$-rays to radio waves and even to gravitational waves, are nicely presented and help in understanding how some more exotic instruments operate.

An author's view of a field is necessarily influenced by background and experience. Hence, Andersen's assertion that "In 1991 the US Air Force decided to declassify the existence of adaptive optics. Overnight, the field of astronomy took a giant leap forward unlike any since the development of mirrored optics" might have been couched differently by civilian French and American astronomers and by technologists who independently developed highly productive astronomical adaptive systems in the 1980s. Likewise, some might question his estimate that "Military space technology tends to be 10-20 years ahead of that in the civilian world".

In a quite different vein, the author's opinion that "Antoine de Saint-Exupéry's accomplishments [as a surveillance pilot] are overshadowed by a children's book he wrote which is widely used to take the fun out of learning French" will sadden many who have been charmed by Le Petit Prince - and it is not relevant to the understanding of telescopes by laypersons.

Andersen's fine piece of work is enlightening and interesting. Experts can dispense with a few chapters that beginners will find useful, along with the appendices on mathematical notation, electromagnetic radiation and "Getting your own telescope". The text flows smoothly, enhanced by vivid discussion and pedagogical flair. And the author's take on remote sensing and surveillance confers a unique feature that will fascinate readers.

René Racine is professor emeritus in the Department of Physics at the Université de Montréal, PO Box 6128, Station Centre-Ville, Montréal, Québéc H3C 3J7, Canada. 